\title{
AVALIAÇÃO DE DEPRESSÃO, SÍNDROME DE BURNOUT E QUALIDADE DE VIDA EM BOMBEIROS
}

\section{Assessment of Depression, Burnout and Quality of Life in Firefighters}

\author{
Makilim Nunes Baptista ${ }^{1}$ \\ Paulo Rogério Morais ${ }^{2}$ \\ Nágila Candido do Carmo ${ }^{3}$ \\ Ginaldo Oliveira de Souza ${ }^{3}$ \\ Alessandra Ferreira da Cunha ${ }^{4}$
}

\section{Resumo}

A avaliação de burnoutem diversas profissões que lidam diretamente com o so frimento humano vem gerando um corpo de conhecimento mais coeso sobre este construto nos últimos anos, no entanto, poucas são as pesquisas que avaliam a associação de burnout com patologias relacionadas ao humor, bem como com a qualidade de vida na cultura brasileira. O objetivo desta pesquisa foi correlacionar sintomatologia depressiva, burnout e qualidade de vida em 101 bombeiros de uma região do interior de São Paulo. Para tanto, foi utilizado um questionário de identificação, contendo dados sociodemográficos e de satisfação com diversas variáveis organizacionais, o Inventário de Burnout (MBI), o Inventário de Beck de Depressão (BDI) e o Inventário de Qualidade de Vida (WHOQOL-bref), em grupos de cinco bombeiros, por um período de 60 minutos. Os resultados demonstraram correlações negativas entre depressão e todas as dimensões de qualidade de vida e correlações positivas entre depressão e as escalas de exaustão emocional e despersonalização de burnout. Os resultados são discutidos à luz das pesquisas envolvendo estes três construtos, já que os achados corroboram grande parte da literatura internacional, bem como são discutidas algumas questões referentes à sobreposição de sintomatologias de depressão e burnout.

Palavras-chave: Depressão; Burnout; Qualidade de vida; Bombeiros.

\section{Abstract}

The evaluation of burnout in several occupations that works with the human suffers is to fecunde to engender a coesive knowledment about this construct in the last years, but few researchs evaluate the association about burnout with humorpathologies and quality of life in ourculture. The purpose of this research was to correlate depressive symptomatology with burnout and quality of life in 101 firefighters in three municipal district of São Paulo (Brazil). For this was use an identification questionnaire, Maslach Bumout Inventory, Beck Depression Inventory and World Health Organization Quality of Life in groups of five firefighter, in the time of a hour. The results show negative correlations between depression and all dimensions of quality of life and positive correlations between emotion exhausting and despersonalization with depression. The results are discuss considering this three concepts, because the international literature confirm the data of this research. Another point in discussion is the similar symptomatology between depression and bumout.

Keywords: Depression; Bumout; Quality of Life; Firefighters.

1 Doutor em Psiquiatria e Psicologia Médica. Programa de Pós-Graduação Stricto Sensu em Psicologia da Universidade São Francisco - Itatiba/SP

2 Mestre pelo Departamento de Psicobiologia da Escola Paulista de Medicina - UNIFESP. Universidade Braz Cubas: Universidade Cruzeiro do Sul; Universidade Ibirapuera

3 Psicólogos, Mogi das Cruzes.

4 Psicóloga. Santo André/SP

Endereço para contato: Rua Dr. Miguel Pierrô, 61 - Cidade Universitária II - Campinas - CEP 13083-300.

E-mail: makilim.baptista@saofrancisco.edu.br 


\section{Introduçã o}

Já está bem estabelecido na literatura que tanto o ambiente profissional quanto outros fatores diretamente relacionados ao desempenho profissional podem ser, em muitos casos, fontes geradoras de estresse. Von Onciul (1996) relacionou uma série de estímulos presentes nos mais diferentes ambientes de trabalho que podem contribuir para 0 surgimento de sintomas de estresse. De modo geral, o ambiente de trabalho possui estímulos físicos (como, por exemplo: ruído, temperatura, tarefas repetitivas, entre outros) e psicossociais (medo de punições ou perda do emprego, alegria ou desafio de uma promoção, conflitos interpessoais, competição com colegas, regras de trabalho contraditórias) que podem ser estressantes. Além disso, algumas atividades profissionais podem apresentar particularidades que as tornam mais ou menos estressantes do que outras.

Entre as possíveis conseqüências de se trabalhar sob estresse constante, a síndrome de burnout tem recebido grande atenção por parte de pesquisadores. Benevides-Pereira (2004) cita que o termo burnout foi empregado por Badley no final da década de 60 para se referir ao desgaste profissional, no entanto, somente se tomou popular em meados da década de 1970 com os trabalhos de Freudenberger (Benevides-Pereira \& Garcia, 2004).

O burnout é uma síndrome psicológica em resposta aos estressores crônicos presentes no ambiente profissional e caracterizada por sintomas de exaustão emocional, despersonalização e reduzida satisfação pessoal com o trabalho, associada a sentimentos de incompetência e ineficácia (Jackson \& Maslack, 1981).

Embora exista a crença largamente difundida da ocorrência de burnout entre os profissionais do corpo de bombeiros, ainda existem poucos estudos que forneçam suporte empírico (Ehlert, Heinrichs \& Wagner, 1998; Palmer \& Spaid, 1996). Essa carência de estudos abre espaço para um trocadilho especulando se os pesquisadores não acreditam que os firefighters (combatentes do fogo - bombeiros, em inglês) estariam imunes a uma síndrome caracterizada pela "queima" completa dos recursos emocionais e de enfrentamento do indivíduo.

Baptista, Souza e Xidieh (2001), baseados em uma revisão da literatura, citam que pro- fissionais cujas atividades estão diretamente envolvidas com os problemas humanos geralmente acabam por se envolver intensamente com as dificuldades psicológicas, sociais e físicas das pessoas que são os clientes de seus serviços. Tais profissionais, em meio ao seu trabalho, muitas vezes sujeitos às várias situações de interação e limitações institucionais, tornam-se alvo de estresse crônico. Por este motivo, pode-se afirmar que entre os profissionais que estão mais expostos às situações e estímulos potencialmente estressantes, encontramse os bombeiros. Do mesmo modo, os profissionais do corpo de bombeiros também correm o risco de desenvolver depressão e transtorno de estresse pós-traumático, embora o ânimo destes profissionais seja geralmente bem mais elevado do que em outros grupos profissionais com ocupação semelhante (Guidotti, 1992).

Em um estudo que investigou a prevalência de sintomas do transtorno de estresse póstraumático em um grupo composto por 318 bombeiros de uma cidade do interior da Alemanha, Ehlert, Heinrichs e Wagner (1998) constataram a prevalência de sintomas em cerca de $18 \%$ dos sujeitos, sendo que $46,2 \%$ dos sujeitos apresentavam sintomatologia subclínica.

Profissionais do corpo de bombeiros, assim como policiais, atendentes de primeiros-socorros de ambulâncias e outros profissionais envolvidos com o atendimento pré-hospitar formam um grupo particularmente exposto a altos níveis de estresse em suas atividades laborais (Kommor, Linton \& Webb, 1993; Gabriel \& Liimatainen, 2000; Smith \& Roberts, 2003).

Bursnall et al. (2001) citam que muitos profissionais como, por exemplo, policiais e bombeiros, estão expostos a situações que lhes exige um elevado grau de gerenciamento e controle das suas respostas emocionais, inibindo a expressão de emoções como medo ou tristeza, substituindoas por expressões de coragem e tranqüilidade. Os autores citam o conceito de emotional labour definido por Hochschild em 1983, como o esforço, planejamento e controle necessários para a expressão de emoções esperadas do profissional e adequadas ao ambiente de trabalho (Morris \& Feldman, 1996 citado por Bursnall et al., 2001).

Uma outra característica que contribui para o surgimento de sintomas de estresse entre os bombeiros é a alternância entre longos períodos de relativa inatividade e situações de alarme 
com momentos de extrema atividade como, por exemplo, os resgates (Guidotti, 1992).

Além disso, esses profissionais freqüentemente se defrontam com situações incontroláveis e que, muitas vezes, ameaçam suas próprias vidas, nas quais decisões e ações rápidas são exigidas (Goldberg et al., 2003). Carlson (1995) cita que a variável mais relevante para determinar se uma situação será ou não estressante é o grau de controle que o sujeito julga ter sobre tal situação. Estudos mostram que, tanto em animais de laboratório quanto no ser humano, os indicadores fisiológicos do estresse são atenuados quando os sujeitos podem exercer algum controle sobre a situação (Weiss, 1968, Frankenhauser \& Rissler, 1970). Respostas cognitivas também podem ser influenciadas pela falta de controle. Fleming, Baum e Singer (1984) citam que situações incontroláveis podem levar o indivíduo a experimentar sentimentos de ineficácia e incompetência. Em um artigo de revisão, Glass e McKnight (1996) apresentam evidências de que a percepção de controle sobre as tarefas profissionais relaciona-se fortemente com sintomas da síndrome de burnout e de depressão.

Além disso, está bem estabelecido na literatura que a percepção de falta de controle sobre os eventos ambientais tem como efeito a geração da condição de desamparo. Aliás, um dos modelos animais mais utilizados tanto para o estudo da depressão quanto para o estudo das respostas de estresse é o do desamparo aprendido, no qual o sujeito experimental não tem qualquer controle sobre os estímulos aversivos que recebe (Willner, 1990; Barrot et al., 2002).

Embora existam especulações acerca da validade da síndrome de burnout como uma entidade nosológica isolada (Leiter, Maslach \& Schaufeli, 2001), marcadamente pela sua elevada correlação com outros quadros psicopatológicos, como, por exemplo, a depressão (Lautert, 1997a), autores têm avaliado simultaneamente a sintomatologia depressiva e a síndrome de burnout (Glass \& Mcknight, 1996).

De fato, parece ser estreita a relação entre eventos estressantes e a precipitação de transtornos mentais (Garcia, 2002; Guimarães, 1999). De acordo com o DSM-IV-TR (APA, 2002), existe forte relação entre a ocorrência de eventos estressantes e o desencadeamento de sintomas depressivos. Tal relação é consubstanciada por novos achados neurofisiológicos, tais como os efeitos do estresse crônico sobre neurônios hipocampais que regulam o funcionamento do eixo hipotálamo-hipófise-adrenal (Baptista, Morais \& Souza, 2004).

Outra variável que tem sua relação com os sintomas de depressão bastante investigada é a qualidade de vida como, por exemplo, apontam Baptista, A.; Baptista, M e Merlin (2004), relatando que as depressões menores influenciam de forma negativa a qualidade de vida de idosos, podendo esta afirmação ser generalizada para várias etapas do desenvolvimento. O DSM-IV-TR (APA, 2002) cita que os indivíduos com transtornos de humor apresentam reduzido funcionamento cognitivo, físico, social e de papéis. Além disso, os sintomas da depressão podem causar prejuízos no funcionamento social e ocupacional. Estudos mostram que pacientes com transtornos apresentam medidas de qualidade de vida inferiores aos indivíduos controle. Além disso, a qualidade de vida pode ser considerada também como um indicativo do impacto da depressão sobre aspectos cognitivos e comportamentais do indivíduo (Anisman et al., 2002), sendo também uma medida útil, inclusive, na avaliação da eficácia de diferentes modalidades de tratamento que visam a restabelecer a funcionalidade psicossocial de pacientes com transtorno depressivo maior (Mahal et al., 2004).

O presente estudo teve como objetivo avaliar as correlações existentes entre sintomatologia de burnout e de depressão e percepção de qualidade de vida em um grupo de bombeiros.

\section{Metodologia}

\section{Participantes}

A amostra utilizada para realização deste estudo foi composta por 101 homens do Corpo de Bombeiro de três municípios de São Paulo, com média de idade de 34 anos (desvio padrão de 5,8 anos), sendo que a maior parte desses participantes era casada ou amasiada (82,2\%). Quanto à escolaridade, 64,4\% afirmaram ter cursado o ensino médio completo, 9,9\% ensino médio incompleto, 6,9\% superior completo, 9,9\% superior incompleto e o restante $(8,9 \%)$ se dividiu em ensino fundamental completo, ensino fundamental incompleto, pós-graduação e mestrado. Os participantes apresentaram tempo de trabalho médio de 12 anos e 7 meses e eram divididos nas seguintes patentes: 
soldado $(54,3 \%)$, cabo $(28,3 \%)$, sargento $(13 \%)$ e comandante de prontidão - CMT (4,3\%), sendo que cerca de $91 \%$ trabalham em escala.

\section{Materiais}

\section{Questionário de caracteriza ção}

Composto por 16 questões fechadas e uma aberta, que avaliaram dados sociodemográficos e profissionais (idade, estado civil, escolaridade, tempo de profissão, cargo ocupado, relação superior direto e principais colegas de trabalho, nível de satisfação com relação aos treinamentos, equipamentos, benefícios, pró-labore, horário de serviço).

\section{Inventá rio de burnout}

Desenvolvido inicialmente por Jackson \& Maslack (1981), traduzido por Lautert (1997b), o questionário possui 22 questões separadas em 3 grupos. Cada grupo avalia um dos fatores que caracterizam o burnout, ou seja, a exaustão emocional, a despersonalização e o sentimento de insatisfação pessoal.

\section{Inventá rio de Depressão de Beck}

Desenvolvido inicialmente por Beck et al. em 1961, traduzido e padronizado para população brasileira por Andrade \& Gorenstein (1998), o questionário constitui-se de 21 itens que avaliaram a tristeza, pessimismo, sensação de fracasso, falta de satisfação, sensação de culpa, de punição, autodepreciação, auto-acusações, idéias suicidas, crises de choro, irritabilidade, retraimento social, indecisão, distorção da imagem corporal, inibição para o trabalho, distúrbio do sono, fadiga, perda de apetite, perda de peso, preocupação somática e diminuição da libido.

\section{Escala de Avaliação da Qualidade de Vida Whoqol-bref}

O WHOQOL-bref é uma versão abreviada e traduzida para o português por Fleck et al. (1999), desenvolvida pelo Grupo de Qualidade de Vida da Organização Mundial de Saúde (OMS), sendo composta de 26 questões que avaliam quatro aspectos da qualidade de vida: físico, psicológico, relações sociais e meio ambiente.

\section{Procedimentos}

Os instrumentos foram aplicados em pequenos grupos (média de 05 bombeiros por grupo), em uma sala de reunião, do próprio destacamento. Esse procedimento foi necessário a fim de se evitar desfalque na prontidão dos grupos no socorro de emergências. A média de tempo de resposta foi de 60 minutos, sendo que os questionários foram aplicados em ordens diferentes, sempre tendo como primeiro o questionário de caracterização, para evitar um possível viés de fadiga.

\section{Resultados}

Os resultados sociodemográficos são expressos de forma descritiva e, para a análise das correlações, se utilizou o teste de Pearson, adotando-se como nível de significância 5\%.

Mediante análise dos resultados de caracterização da amostra, observou-se que quando não estão trabalhando na corporação, $74,3 \%$ dos participantes fica junto da família, $25,7 \%$ assiste programas de televisão, $14,9 \%$ pratica atividade física, 8,9\% lê livros e/ ou estuda, 6,9\% lê jornais e revistas, $2,0 \%$ vai ao cinema e $8,9 \%$ pratica outras atividades.

Quanto à questão da satisfação profissional, expressos na tabela 1 , os participantes apresentaram maiores freqüências de satisfação (totalmente/parcialmente satisfeitos) com relação aos principais colegas, treinamentos, superior direto, equipamentos e escala de trabalho enquanto que apresentaram menores freqüências de satisfação em relação aos benefícios e salários. 
Tabela 1 - Satisfação profissional

\begin{tabular}{l|l|l|l|l}
\hline & $\begin{array}{l}\text { Totalmente } \\
\text { Satisfeito (\%) }\end{array}$ & $\begin{array}{l}\text { Parcialmente } \\
\text { Satisfeito (\%) }\end{array}$ & $\begin{array}{l}\text { Parcialmente } \\
\text { Insatisfeito (\%) }\end{array}$ & $\begin{array}{l}\text { Totalmente } \\
\text { Insatisfeito (\%) }\end{array}$ \\
\hline Principais Colegas & 50,5 & 37,6 & 6,9 & 5,0 \\
Treinamentos & 25,7 & 54,5 & 8,9 & 10,9 \\
Superior Direto & 51,0 & 41,0 & 4,0 & 4,0 \\
Equipamentos & 25,7 & 50,5 & 21,8 & 2,0 \\
Benefícios & 11,9 & 36,6 & 25,7 & 25,7 \\
Salários & 6,9 & 54,5 & 24,8 & 13,9 \\
Escala & 36,3 & 6,6 & 6,6 \\
\hline
\end{tabular}

Com relação à sintomatologia depressiva, $81,18 \%$ dos participantes apresentou escores não significativos, 13,86\% com nível leve, 3,96\% nível moderado e apenas 1\% apresentou nível severo de sintomatologia depressiva. As correlações entre sintomatologia depressiva, burnout e qualidade de vida são demonstradas na tabela 2 .

\section{Tabela 2 - Correlações entre depressão, qualidade de vida e burnout}

\begin{tabular}{l|c|c}
\hline Variáveis & r & p \\
\hline Depressão X WHOQOL domínio 1 (Físico) &,- 522 &, 000 \\
Depressão X WHOQOL domínio 2 (Psicológico) &,- 558 &, 000 \\
Depressão X WHOQOL domínio 3 (Relações Sociais) &,- 568 &, 000 \\
Depressão X WHOQOL domínio 4 (Meio Ambiente) &,- 535 &, 000 \\
Depressão X MBI (Exaustão Emocional) &, 512 &, 000 \\
Depressão X MBI (despersonalização) &, 301 &, 002 \\
\hline
\end{tabular}

Podem-se observar correlações negativas entre a sintomatologia depressiva e os quatro domínios da qualidade de vida e correlações positiva entre a sintomatologia depressiva com as dimensões de exaustão emocional e despersonalização de burnout. Esses resultados podem ser expressos considerando que quanto maior a sintomatologia depressiva, menores os níveis de percepção de qualidade de vida e, quanto maior os níveis de sintomatologia de depressão, maiores os escores em exaustão emocional e despersonalização no construto burnout.

\section{Discussão}

Os bombeiros estão sujeitos não só a elementos que podem ter efeitos adversos sobre seu organismo, como gases e substâncias tóxicas, temperaturas elevadas, materiais perfuro-cortantes, entre outros (Landrigan et al. 2004), mas também a situações potencialmente traumáticas como acidentes envolvendo crianças, pessoas com queimaduras ou ferimentos graves (Smith \& Roberts, 2003), corpos mutilados e cenas de destruição material (Wagner, Heinrichs \& Ehlert, 1998).

Pode-se observar nesta amostra uma satisfação com a maioria das situações relacionadas ao trabalho, tais como o relacionamento com os colegas e superior direto, treinamento oferecido, equipamentos e sistema de escalonamento, o que vem corroborar com os achados de Kalimo et al. (1980) observando, em uma amostra composta por 260 bombeiros, que estes profissionais estavam satisfeitos com seu trabalho, o que se associou com raros sinais de problemas psicológicos crônicos.

A respeito da freqüência de sintomatologia depressiva, é interessante notar que aproximadamente $19 \%$ dos profissionais apresentaram sintomatologia clinicamente significativa de depressão, o que não representa diagnóstico de depressão, devendo-se avaliar a possibilidade de sobre- 
posição de sintomas entre depressão e burnout (Baba, Galperin \& Lituchy, 1999; Glass \& Mcnight, 1996)

Em seu artigo de revisão, Glass \& McNight (1996) também apresentam estudos nos quais foram observadas correlações positivas entre a sintomatologia depressiva e a síndrome de burnout, nas dimensões de exaustão emocional e despersonalização, e correlações negativas na dimensão de realização pessoal, o que confirma parcialmente os dados deste estudo.

Da mesma forma, Arice e cols. (2004), ao avaliarem correlações entre burnout e sintomatologia depressiva em um grupo de funcionários públicos brasileiros, também encontraram correlações positivas entre sintomatologia depressiva e burnout (em mulheres) muito próximas das encontradas neste estudo. No estudo de Arice e cols. (2004) encontrou-se, além das correlações entre BDI e Desgaste Emocional $(\mathrm{r}=0,409)$; BDI e Despersonalização $(\mathrm{r}=0,279)$, também encontrou-se correlação com Incompetência $(r=0,268)$, também corroborando parcialmente os achados desta pesquisa.

Uma outra questão que deve ser avaliada com a devida cautela diz respeito à possibilidade de sobreposição de sintomas entre a depressão e o burnout como, por exemplo, os sintomas de rebaixamento motor. Glass e McKnight (1996), em seu artigo de revisão, apontam para o questionamento de sobreposição de alguns sintomas, apesar de oferecerem evidências de que se tratam de construtos diferentes. Os mesmos autores ainda apontam a falta de modelos explicativos mais complexos para avaliar a relação entre os dois fenômenos.

É importante frisar que o burnout pode desencadear sintomatologia depressiva, sendo 0 inverso também verdadeiro, no entanto, Baba, Galperin e Lituchy (1999) enfatizam que o burnout mais comumente precederia o desenvolvimento de sintomatologia depressiva.

A bibliografia não aponta a relação entre burnout e qualidade de vida ou bem-estar psicológico, como citado por Maslach (2001), no sentido de facilitar comparações com os resultados aqui expressos, no entanto, parece lógica a relação entre estes dois construtos já que a qualidade de vida avalia a maneira que o indivíduo percebe a sua posição na vida, no seu contexto cultural e sistema de valores nos quais ele vive e relacionados às suas expectativas, seus objetivos, padrões e preocupações. Se a relação com o trabalho não é considerada como satisfatória, é esperado que o indivíduo também avalie sua qualidade de vida relacionada a este aspecto laboral de forma correlata, principalmente porque grande parte das horas semanais é vivida no trabalho.

Dessa forma, se tornam fundamentais constantes avaliações de saúde mental, qualidade de vida e burnout em trabalhadores de diversos setores e atividades, pois somente desta forma se podem desenvolver, caso detectados altos níveis de depressão e estresse organizacional, formas eficazes de intervenção, a fim de proporcionar a melhoria das habilidades de enfrentamento das situações relacionadas com o clima laboral, como recomendam Souza, Baptista e Xidieh (2001). Também se fazem necessários estudos longitudinais capazes de hipotetizar modelos explicativos e fatores de risco envolvidos com a associação entre sintomatologia depressiva e burnout, a fim de se formar um corpo de estudos mais concatenados e explicativos entre esses fenômenos.

\section{Referências}

American Psychiatric Association (2002). Manual Diagnóstico e Estatístico dos Transtornos Psiquiátricos. (4a ed revisada). Trad. Claúdia Dornelles. Porto Alegre: Artmed.

Andrade, L \& Gorenstein, C. (1998). Inventário de depressão de Beck; Propriedades psicométricas da versão em português. Revista de Psiquiatria Clinica. 25 (5), 1998. Recuperado em 30 abri 2002: http// w w w.hcnet.usp.br/ip q/ revista/r255/ depre255b.htm.

Anisman, H. et al. (2002). Stress, coping, uplifts, and quality of life in subtypes of depression: a conceptual frame and emerging data. Affect Disord, 71 (1), 121-130.

Arice, G. et al. (2004). Correlação entre Sintomatologia Depressiva e Burnout em um Grupo de Servidores Públicos. Psicologia Argumento, 22 (37), 53-62. 
Baba, V.V., Galperin, B.L \& Lituchy, T. R. (1999). Occupational Mental Health: a study of work-related depression among nurses in the Caribbean. International Journal of Nursing Srudies, 36, 163-169.

Baptista, A. S. D.; Baptista, M. N. \& Merlin, M. S. (2004). Depressão e Suicídio na Terceira Idade. In Baptista, M. N. Suicídio e depressão: atualizações. Rio de janeiro: Guanabara-Koogan.

Baptista, M. N.; Morais, P. R.\& Souza, F. G. (2004). Relações entre síndrome de burnout e transtornos de humor. In Baptista, M. N. Suicídio e depressão: atualizações. Rio de Janeiro: Guanabara Koogan.

Baptista, M. N, Souza, F. G.\& Xidieh, G. F. (2001). Burnout: definição e características. Infanto - Rev. Neuropsiq. da Inf. e Adol., 9(3), 100-102.

Barrot, M. et al. (2002). Neurobiology of depression. Neuron, 34, (1), 13-25.

Benevides-Pereira, A. M. T. (2003). O estado da arte do burnout no Brasil. InterAção Psy, 1: 4-11. Recuperado em 30 ago 2004: http:// www.dpi.uem.br/Interacao/Numero\%201/PDF/ Artigos/Artigo1.pdf

Benevides-Pereira, A. M. T. \& Garcia, L P. (2003). Investigando o burnout em professores universitários. InterAção Psy, 1, 76-89. Recuperado em 30 ago 2004: http://www.dpi.uem.br/Interacao/ Numero\%201/PDF/Artigos/Artigo 6.pdf.

Berkowitz, G. et al. (2004). Health and environmental consequences of the world trade center disaster. Environ Health Perspect, 112 (6), p731739 .

Bursnall, S et al. (2001). Occupational Stress: A review of factors influencing its occurrence and effective management. WorkCover: Shenton Park.

Carlson, N.R.(1995). Foundations of physiological psychology. (3a ed.). Allyn and Bacon: Boston, 1995.

Daleva, M. et al. (1980). Psychological and biochemical strain in firemen's work. Scand J Work Environ Health, 3, 179-187.

Ehlert, U.; Heinrichs, M.\& Wagner, D. (1998). Prevalence of symptoms of posttraumatic stress disor- der in German professional firefighters. Am J Psychiatry, 155 (12), 1727-1732.

Fleck, M. P. A. et al. (1999). Desenvolvimento da versão em português do instrumento de avaliação de Qualidade de Vida da OMS (WHOQOL-100). Revista Brasileira de Psiquiatria, 21 (1), 19-28.

Fleming, R.; Baum, A.\& Singer, J.E. (1984). Toward and integrative approach tho the study of stress. J. Pers. Soc. Psychol., 46 (4), 939-949.

Frankenhaeuser, M. \& Rissler, A. (1970). Effects of punishment on catecholamine release and efficiency of performane. Psychopharmacologia, 17(5), 378-390.

Gabriel, P. \& Liimatainen, M.R. (2000). Mental health in the workplace: introduction executive summaries. International Labour Office: Geneva.

Garcia, R. (2002). Stress, synaptic plasticity, and psychopathology. Rev Neurosci, 13 (3), 195-208.

Glass, D. C. \& Mcknight, J. D. (1996). Perceived control, depressive symptomatology and professional burnout: a review of the evidence. Psychology and Health, 11, 23-48.

Goldberg, G. et al. (2003). Postmortem inquiries and trauma responses in paramedics and firefighters. J Interpers Violence, 18 (6), 607-622.

Guidotti, T. L. (1992). Human factors in firefighting: ergonomic, cardiopulmonary, and psychogenic stress-related issues. Int Arch Occup Environ Health, 64 (1), 1-12.

Guimarães, F.S. (1999). Transtornos afetivos. In: Brandão, M. L \& Graeff, F. G. Neurobiologia das doenças mentais. (5a ed.) Lemos: São Paulo.

Jackson, S. E. \& Maslach, C. The Measurement of Experienced Burnout. Journal of Occupational Behaviour, 2, 99-113.

Kommor, M. J., Linton, J. C. \& Webb, C. H. (1993). Helping the helpers: the development of a critical incident stress management team through university/community cooperation. Ann Emerg Med., 22 (4), 663-8.

Lautert, L. (1997a) Desgaste Profissional: Uma Revisão da Literatura e Implicações para a Enfermaria. Revista Gaúcha Enfermagem, 18 (2), 83-93. 
Lautert, L (1997b). O desgaste profissional: estudo empírico com enfermeiras que trabalham em hospitais. Revista Gaúcha de Enfermagem, 18 (2), 133-144.

Leiter, M. P., Maslach, C.\& Schaufeli, W. B. (2001). Job burnout. Annu. Rev. Psychol., 32, 397-422.

Mahal, Y. et al. (2004). Quality of life assessments in major depressive disorder: a review of the literature. Gen Hospital Psychiatry, 26, (1), 13-17.

Maslach, C. (2001). What have you learned about burnout and health? Psychology and Health, 16, 607-611.

Palmer, R.G. \& Spaid, W. M. (1996). Authoritarianism, inner/ other directedness, and sensation seeking in firefighter/paramedics: their relationship with burnout. Prehospital Disaster Med, 11 (1), 11-5.
Roberts, K. \& Smith, A. (2003). Interventions for post-traumatic stress disorder and psychological distress in emergency ambulance personnel: a review of the literature.Emergency Med Journal, 20 (1), 75-78.

Von Onciul, J. (1996). ABC of Work Related Disorders: Stress at work. BMJ, 313(7059), 745748.

Weiss, J. M. (1968). Effects of coping response on stress. Journal of Comparative Physiological Psychology, 65 (1) 251-260.

Willner, P. (1990). Animal models of depression: an overview. Pharmacology and Therapeutics, 45 (3), 425-455.

Recebido em/Received in: 28/03/2005 Aprovado em/Approved in: 05/05/2005 